\title{
Um modelo experimental de ablação do Sistema Nervoso Intrínseco Cardíaco reduz a contratilidade do coração de ratos
}

\author{
A new experimental model of chemical ablation of the Intrinsic Cardiac Nervous System reduces \\ heart contractility and causes a type of dilated cardiopathy in rats
}

Adilson SCORZONI FILHO, Ernani J. NAKAMURA, Sandro Mendonça FARIA, Alexandre Henrique MARCHETTI, José Mário BRANDÃO, Alexandre Luiz ARANHA, Luis Augusto MATTAR, Walter Vilella Andrade VICENTE, Sérgio Britto GARCIA

RBCCV 44205-700

\section{Resumo}

Objetivo: A função do Sistema Nervoso Intrínseco Cardíaco e o seu papel na doença cardíaca permanecem pobremente compreendidos. Sabe-se que o cloreto de benzalcônio (CB) induz a desnervação intrínseca do tubo digestivo. $O$ objetivo deste estudo foi tentar produzir um modelo experimental de desnervação intrínseca do coração utilizando o CB.

Método: Trinta ratos Wistar foram submetidos à aplicação intrapericárdica de $C B(0,3 \%)$ e trinta animais controle receberam a solução salina. Após 15 dias, os animais foram divididos em três grupos, com 10 animais tratados e 10 controles em cada. Os animais do grupo I foram submetidos a estudo radiológico e histopatológico. A área cardíaca e o índice cardiotorácico (ICT) foram medidos nas radiografias. Os animais do grupo II foram submetidos a estudo hemodinâmico com registro da pressão arterial, freqüência cardíaca e débito cardíaco. No grupo III, a integridade da inervação parassimpática extrínseca do coração foi avaliada por estimulação vagal direita. $O$ sistema de condução foi avaliado pelo ECG basal.

Resultados: A aplicação de CB acarretou aumento do ICT, da área cardíaca, pressão arterial e débito cardíaco, bem como do peso ponderal e do fígado. Nestes animais, a análise histopatológica mostrou redução do número de neurônios atriais e congestão passiva crônica do fígado. A estimulação vagal não mostrou diferenças entre os grupos experimentais.

Conclusão: A ablação do sistema nervoso intrínseco propiciou o aparecimento de cardiopatia dilatada com insuficiência cardíaca direita e esquerda. Esse modelo experimental inédito deverá nortear futuros estudos na tentativa da elucidação da relação entre lesão neuronal e miocardiopatia.

Descritores: Contração miocárdica. Sistema de condução cardíaco. Sistema nervoso autônomo. Gânglios parassimpáticos. Denervação autônoma. Compostos de benzalcônio. Miocardiopatia congestiva. Insuficiência cardíaca congestiva.

Trabalho realizado pelo Departamento de Patologia, Divisão de Cirurgia Torácica e Cardiovascular do Departamento de Cirurgia e Anatomia e Departamento de Fisiologia da Faculdade de Medicina de Ribeirão Preto da Universidade de São Paulo, Ribeirão Preto, SP.

Endereço para correspondência: Prof. Dr. Sérgio Britto Garcia. Departamento de Patologia - Faculdade de Medicina de Ribeirão Preto - Campus USP. Av.Bandeirantes, 3900. Ribeirão Preto, SP CEP 14.040-900. E-mail: sbgarcia@fmrp.usp.br 


\section{Abstract}

Objective: The function of Intrinsic Cardiac Nervous System is largely unknown, as is its role in heart disease. In the digestive system, a topic aplication of Benzalkonium chloride (BC) leads to intrinsic denervation of the viscera. Thus, our aim was to obtain an experimental model of cardiac intrinsic denervation by the application of $\mathrm{BC}$

Method: Thirty male Wistar rats received intrapericardic injection of CB $0.3 \%$ (CB animals) and thirty similar animals received saline ( $\mathrm{C}$ animals). After 15 days the animals were divided in three groups, with $10 \mathrm{CB}$-treated and 10 salinetreated animals each. Group I was submitted to radiological and morphologic studies. The cardiac shadow area (CSA) and cardiothoracic index were calculated in roentgenograms with a semi-automatic image analysis system (MINI-MOP) The day after the animals were weighted and sacrificed with heart, liver and lung collected for histopathologic analysis. The animals of group II were submitted to a hemodynamic study. Measurements of blood pressure, heart rate and cardiac output were performed using the Cardiomax II termodilution system and a Termistor sensor. With the animals of the group III, the integrity of extrinsic parassympatic cardiac innervartion was examined by measuring heart rate response

\section{INTRODUÇ̃̃O}

Os neurônios intrínsecos cardíacos haviam sido classicamente considerados como sendo somente neurônios pós-ganglionares parassimpáticos eferentes que recebem impulsos dos neurônios pré-ganglionares eferentes parassimpáticos, com os gânglios intrínsecos funcionando como estações transmissoras cardíacas dos impulsos do sistema nervoso central. Posteriormente, foram identificados neurônios eferentes envolvidos num circuito local de interconexão que continha também neurônios eferentes pós-ganglionares simpáticos e parassimpáticos, bem como aferentes [1], sendo todo este conjunto chamado de Sistema Nervoso Intrínseco Cardíaco (SNIC). Contudo, a função do SNIC e seu papel na fisiopatologia cardíaca permanecem pobremente compreendidos. Os inúmeros modelos experimentais descritos de desnervação extrínseca do coração [2-4], colaboraram, em parte, para a compreensão da neurocardioregulação.

Todos estes modelos demonstraram desnervação cardíaca, mas os autores não levaram em consideração um fato importante da desnervação do transplante cardíaco, a qual não inclui desnervação intrínseca, porque os gânglios intracardíacos não são afetados nesse tipo de procedimento. De fato, enquanto os enxertos cardíacos humanos permanecem funcionando extrinsecamente desnervados, eles apresentam viabilidade dos nervos intrínsecos [5] e as atividades dos plexos ganglionares intrínsecos cardíacos retornam [6]. Desta forma, não há descrição na literatura de um modelo experimental específico de desnervação do SNIC, preservando o sistema extrínseco. to electrical stimulation of the right vagus. Electrical activity was assessed by ECG

Results: CB animals presented increases in cardiothoracic index, CSA, body and liver weight. In these animals the histopathologic analysis showed passive chronic congestion and reduction of the number of atrial neurons. In the hemodynamic study, total peripheral resistance and heart rate were similar in both groups, but blood pressure and cardiac index were reduced in the $C B$ group. The vagal stimulation and ECG were similar in both groups.

Conclusion: The Intrinsic Cardiac Nervous System denervation caused dilated cardiopathy in rats with left and right heart failure. The etiology of some dilated cardiopathies in human is largely unknown. Thisunpublished experimental model should provide future studies with the objective of elucidating the relationship between neuronal injures and heart disease.

Descriptors: Myocardial contraction. Heart conduction system. Autonomic nervous system. Ganglia, parasympathetic. Autonomic denervation. Benzalkonium compounds. Cardiomyopathy, congestive. Heart failure, congestive.

O cloreto de benzalcônio (CB) é um surfactante catiônico que, aplicado topicamente no tubo digestivo, causa desnervação dos neurônios intrínsecos (mioentéricos), com conseqüentes alterações importantes na fisiologia e morfologia desses órgãos $[7,8]$. Como os neurônios cardíacos localizam-se no epicárdio [9], por analogia, a administração intrapericárdica de CB poderia destruí-los, produzindo um modelo experimental de desnervação. A proposta desse estudo é, portanto, determinar os efeitos da ablação do SNIC na morfologia, contratilidade e freqüência cardíaca de ratos, como forma de melhor entender o papel do SNIC na fisiopatologia cardíaca.

\section{MÉTODO}

\section{Animais}

Foram utilizados 60 ratos Wistar, machos, com $200 \mathrm{~g}$ de peso corporal no início do experimento. Todos os animais receberam cuidados humanos em conformidade com "Principles of Laboratory Animal Care", formulados pela National Society for Medical Research, e com "Guide for the Care and Use of Laboratory Animals", publicado pelo National Institutes of Health (NIH publicação 86-23, revisado 1985).

\section{Grupos e Desenho Experimental}

Trinta ratos Wistar foram submetidos à aplicação intrapericárdica de $\mathrm{CB}(0,3 \%)$ e 30 animais controle receberam a solução salina. Após 15 dias, os animais foram divididos em três grupos, com 10 animais tratados e 10 controles em cada. Os animais do grupo I foram submetidos 
a estudo radiológico e histopatológico. A área cardíaca e o índice cardiotorácico (ICT) foram medidos em radiografias. Os animais do grupo II foram submetidos a estudo hemodinâmico com registro da pressão arterial, freqüência cardíaca e débito cardíaco. No grupo III, a integridade da inervação parassimpática extrínseca do coração foi avaliada por estimulação vagal direita. $\mathrm{O}$ sistema de condução foi avaliado pelo ECG basal.

\section{Desnervação intrínseca do coração}

Após anestesia por inalação de éter sulfúrico em câmara fechada, foi realizada laparotomia transversa de aproximadamente $4 \mathrm{~cm}$ no abdome superior e com uma seringa e agulha $(25 \mathrm{G})$ foi puncionado o saco pericárdico no centro tendíneo do diafragma e injetado $0,2 \mathrm{ml}$ of $\mathrm{CB}$ a $0,3 \%$. O fechamento do plano muscular e cutâneo foi realizado com sutura contínua de algodão 2-0. Outro grupo (animais controle) recebeu solução de cloreto de sódio a $0,9 \%$ no saco pericárdico.

\section{Estudo radiológico}

Os ratos foram submetidos a exame radiológico simples de tórax na incidência PA, com aparelho de radiografia odontológica encostado ao dorso do animal. Foram avaliados: a área cardíaca, o diâmetro látero-lateral cardíaco e torácico através da medida nas radiografias por um sistema semiautomático de análise de imagens (MINI-POP, Kontron®). Foi também calculado o índice cardiotorácico (ICT).

\section{Estudo histopatológico}

No dia seguinte ao exame radiológico, os animais apresentavam bom estado geral e foram pesados, sendo em seguida sacrificados com infusão endovenosa de Cloreto de Potássio ( $1 \mathrm{meq} / \mathrm{ml})$, com objetivo de induzir uma parada cardíaca em diástole. Na seqüência, eram retirados e pesados coração e fígado e coletado fragmento do lobo inferior direito do pulmão e encaminhados para rotina histológica. Foram realizados 15 cortes seriados transversais dos átrios de cada animal, padronizadamente, para quantificar o número de neurônios intrínsecos cardíacos, como descrito por OLIVEIRA et al. [8].

Com o intuito de verificar a especificidade do CB para desnervação, em um grupo de cinco animais foi feito o mesmo procedimento para injeção intrapericárdica de carraginina, uma substância sabidamente indutora de reação inflamatória. Os corações destes animais foram submetidos ao mesmo estudo histopatológico descrito acima.

Estudo eletrocardiográfico e de estimulação elétrica vagal

A integridade da inervação extrínseca parassimpática do coração foi examinada pela medida da resposta da freqüência cardíaca à estimulação elétrica do nervo vago direito. A freqüência e a integridade do sistema de condução cardíaco foram avaliadas por eletrocardiografia (ECG).

Três eletrodos foram implantados no subcutâneo do dorso de cada rato e conectados a um gravador multicanal (Physiograph Four-A, E \& M Instrument Company, New York, NY). A morfologia do ECG e o decréscimo da freqüência cardíaca produzida pela estimulação elétrica do nervo vago direito foram estudados em ratos anestesiados com cloralose endovenoso. O intervalo entre as estimulações foi determinado pelo tempo requerido para freqüência cardíaca voltar ao nível de pré-estimulação.

\section{Estudo hemodinâmico}

Este experimento consistiu em aferir hemodinamicamente as respostas obtidas após desnervação intrínseca do coração e, desse modo, estudar sua contratilidade. Cânulas de polietileno foram introduzidas na artéria femoral direita, e veia jugular direita, sob anestesia intraperitoneal com nembutal e o sensor de temperatura Termistor (Columbus Instruments) foi inserido na artéria carótida comum direita até a crossa da aorta. Um fluxômetro de termodiluição Cardiomax II 85 (Columbus Instruments) foi usado para realizar as medidas. Essas medidas foram aferidas 15 minutos após o procedimento cirúrgico ou quando os parâmetros hemodinâmicos estivessem estabilizados.

A pressão arterial média (PAM) e a freqüência cardíaca (FC) foram medidas pelo fluxômetro, por meio da cânula da artéria femoral. O volume sistólico (VS) foi medido usando curvas de termodiluição. Por meio da cânula jugular, solução de $\mathrm{NaCl} 0,9 \%$ em baixa temperatura foi injetada com Micro Injector 400 (Columbus Instruments) e o Termistor captava a variação de temperatura.

VS foi calculado pela fórmula: (Tb-Ti) $x V i$; onde: VS - Volume $A S C$

Sistólico, Tb- temperatura sanguínea, Ti- Temperatura da Injeção, Vi- Volume injetado, ASC-Área Sob a Curva. Os parâmetros seguintes foram calculados:

- DCM (Débito Cardíaco Minuto): DCM = FC x VS

- IC (Índice Cardíaco): $I C=D C M$

$100 \mathrm{~g}$

- IS (Índice Sistólico): $I S=V S$ $100 \mathrm{~g}$

- RVPT (Resistência Vascular Periférica Total): $R V P T=P A M$ $D C M$

\section{Análise estatística}

Os resultados foram apresentados como média \pm desvio 
padrão. $O$ teste $t$ de Student foi utilizado para a análise comparativa, com nível de significância estabelecido em 5\%.

\section{RESULTADOS}

O estudo radiológico mostrou aumento da área cardíaca e do índice cardiotorácico no grupo $\mathrm{CB}$ em relação ao controle (Tabela 1). Observamos aumento do peso médio ponderal do fígado no grupo $\mathrm{CB}$, quando comparado ao controle, sem alteração do peso do coração (Tabela 2).

Tabela 1. Resultados das análises radiológicas, com imagens obtidas pelo estudo radiológico em projeção pósteroanterior.

\begin{tabular}{lll}
\hline & Grupo C & Grupo CB \\
\hline Índice cardiotorácico & $0,34 \pm 0,02$ & $0,42 \pm 0,08^{*}$ \\
Área cardíaca $(\mathrm{mm} 2)$ & $204,61 \pm 15.05$ & $283,37 \pm 33,63^{*}$ \\
\hline
\end{tabular}

$* \mathrm{CB}>\mathrm{C}(\mathrm{p}<0,05)$

Tabela 2. Pesos corporal, cardíaco e hepático.

\begin{tabular}{lll}
\hline & Grupo C & Grupo CB \\
\hline Peso do fígado $(\mathrm{g})$ & $9,78 \pm 1,03$ & $11,64 \pm 1,28^{*}$ \\
Peso do coração $(\mathrm{g})$ & $1,01 \pm 0,09$ & $1,04 \pm 0,11$ \\
Peso corporal $(\mathrm{g})$ & $242,20 \pm 15,73$ & $274,09 \pm 23,98^{*}$
\end{tabular}

$* \mathrm{CB}>\mathrm{C}(\mathrm{p}<0,05)$

O estudo histopatológico mostrou que os corações do grupo controle eram normais e que, no grupo $\mathrm{CB}$, os corações apresentavam necrose nos plexos ganglionares, áreas de hipertrofia miocárdica alternadas com áreas de hipotrofia. Foi observado discreto espessamento do epicárdio no grupo $\mathrm{CB}$, mas nenhuma adesão importante ou atividade inflamatória aguda foi notada. Nos corações de ratos tratados com carraginina observou-se discreto espessamento pericárdico e ausência de outras alterações histopatológicas, sendo que houve preservação dos neurônios intrínsecos do coração.

Nos fígados do grupo CB observou-se presença de congestão passiva crônica caracterizada por dilatação da veia centrolobular acompanhada de dilatação sinusoidal . Não foram observadas alterações histopatológicas nos fígados dos animais dos grupos controle e carraginina. Os pulmões estavam histologicamente normais em todos os grupos. A contagem neuronal mostrou diminuição do número de neurônios cardíacos nos animais do grupo $\mathrm{CB}$ em relação ao grupo $\mathrm{C}$ (Tabela 3 ).

O estudo hemodinâmico (Tabela 4) mostrou que os ratos do grupo CB apresentaram diminuição da PAM, DC, VS e IC, em comparação ao grupo controle. A freqüência cardíaca e a resistência vascular periférica total não apresentaram alterações significativas, quando comparadas ao grupo controle.

Tabela 3. Resultados da contagem neuronal.

\begin{tabular}{lll}
\hline & Grupo C & Grupo CB \\
\hline $\begin{array}{l}\text { Número de neurônios atriais } \\
\text { (por } 100 \mu \mathrm{m} \text { de miocárdio) }\end{array}$ & $380,90 \pm 44,75$ & $112,51 \pm 21,28^{*}$ \\
\end{tabular}

* $\mathrm{CB}<\mathrm{C}(\mathrm{p}<0,05)$

Tabela 4. Resultados do estudo hemodinâmico, em animais dos grupos C e CB.

\begin{tabular}{lll}
\hline & Grupo C & Grupo CB \\
\hline $\begin{array}{l}\text { Freqüência cardíaca } \\
\text { (batimentos por min) }\end{array}$ & $405 \pm 30$ & $429 \pm 39$ \\
$\begin{array}{l}\text { Pressão arterial média } \\
(\mathrm{mmHg})\end{array}$ & $117 \pm 5$ & $96 \pm 3^{*}$ \\
$\begin{array}{l}\text { Débito cardíaco }(\mathrm{mcl} / \mathrm{min}) \\
\text { Volume sistólico }(\mu \mathrm{l} / 100 \mathrm{~g})\end{array}$ & $137 \pm 9$ & $101 \pm 6^{*}$ \\
Índice cardíaco $(\mathrm{mcl} / \mathrm{min} / \mathrm{g})$ & $0,47 \pm 0,05$ & $0,32 \pm 0,04^{*}$ \\
$\begin{array}{l}\text { Resistência vascular periférica } \\
(\mathrm{mmHg} / \mathrm{mcl} / \mathrm{min})\end{array}$ & $0,87 \pm 0,40$ & $1,00 \pm 0,14$ \\
\hline
\end{tabular}

* $\mathrm{CB}<\mathrm{C}(\mathrm{p}<0,05)$

O ECG mostrou diminuição da variabilidade do intervalo RR no grupo $\mathrm{CB}$, estando todos os demais parâmetros idênticos e dentro do padrão de normalidade. As quedas nas freqüências cardíacas, produzidas pela estimulação elétrica no nervo vago direito, foram semelhantes nos dois grupos (Figura 1).

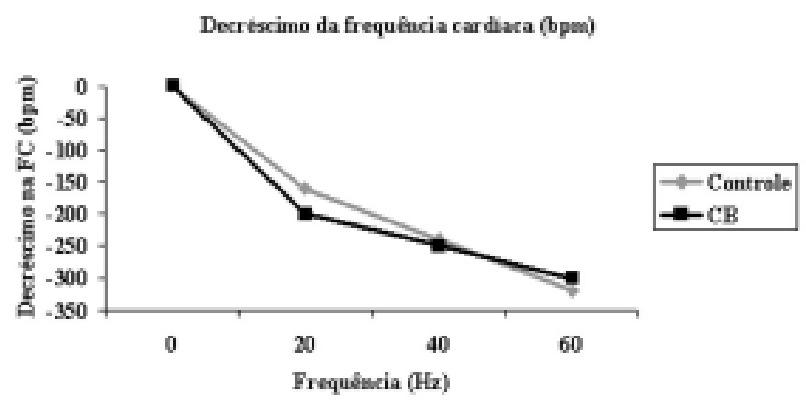

Fig. 1 - Freqüência cardíaca de repouso e resposta bradicárdica à estimulação elétrica vagal direita $(5 \mathrm{~V}, 2 \mathrm{~ms}, 1-128 \mathrm{~Hz})(\mathrm{n}=10, \mathrm{NS})$.

\section{COMENTÁRIOS}

Estudos têm demonstrado que o SNIC fornece estímulo neurohumoral ao coração, modulando freqüência e contratilidade cardíacas [10]. O SNIC atua como um processador 
de distribuição, integrando informações eferentes centrífugas parassimpática e simpática para o coração, além de informações centrípetas vindas das fibras nervosas sensitivas [1].

O papel do SNIC na patogênese cardíaca é desconhecido. Disfunção autonômica é uma complicação séria do diabetes melittus e pode causar disfunção ventricular [11], assim como na cardiopatia chagásica [12]. Uma ferramenta comum e útil para estudar aspectos funcionais de sistema nervoso e inervação visceral é verificar os efeitos de remoção de partes do sistema nervoso. O desenvolvimento de um novo modelo de ablação do SNIC usando o cloreto de benzalcôneo (CB) vem sendo motivo de investigações há algum tempo [7]. Já se comprovou, em ratos, que a administração tópica de $\mathrm{CB}$ na serosa do tubo digestivo produz destruição de neurônios mioentéricos intrínsecos e que este procedimento produz profunda mudança na fisiologia e morfologia do órgão. Assim, é descrito que esse tipo de desnervação leva a importante distensão dessas vísceras com prejuízo da sua motilidade $[12,13]$. Os neurônios cardíacos situam-se sob o epicárdio do coração [10] e por analogia com o modelo digestivo, a administração intrapericárdica de CB poderia destruir esses gânglios, produzindo um modelo experimental de desnervação intrínseca o coração.

Esta foi a idéia fundamental desta investigação, cujos achados principais foram: 1) $\mathrm{O}$ estudo radiológico mostrou que o ICT e a área cardíaca do grupo $\mathrm{CB}$ foram significativamente maiores que o grupo controle; 2) $\mathrm{O}$ grupo $\mathrm{CB}$ apresentou aumento dos pesos ponderal e do figado; 3) A análise histopatológica do figado foi normal no grupo controle e mostrou congestão passiva crônica no grupo $\mathrm{CB}$ e no coração foram observadas áreas de hipertrofia mescladas com áreas de hipotrofia e diminuição da contagem neuronal nos átrios; 4) A estimulação vagal não mostrou alteração significativa no decréscimo da freqüência cardíaca dos grupos, sendo que ao ECG observou-se apenas diminuição do intervalo R-R no grupo CB; 5) No estudo hemodinâmico, a resistência vascular sistêmica e freqüência cardíaca foram semelhantes nos grupos, porém a pressão arterial e o índice cardíaco foram significativamente menores no grupo $\mathrm{CB}$.

A histopatologia mostrou um discreto espessamento do pericárdio, mas não um processo inflamatório ativo que pudesse explicar os demais achados morfológicos e funcionais. Nos animais submetidos à aplicação de carraginina não houve lesões dos neurônios intrínsecos cardíacos, o que mostra a especificidade do $\mathrm{CB}$ para produzir tais lesões.

No modelo experimental desenvolvido, a destruição do SNIC foi parcial e confirmada pela contagem neuronal. Os animais desnervados apresentaram ganho de peso que pode ser causado pela retenção hídrica, usualmente observada na insuficiência cardíaca congestiva.

Concluímos, portanto, que a desnervação do SNIC causa um tipo de cardiopatia dilatada, demonstrada pelo estudo radiológico e sem ganho de massa muscular na comparação dos pesos dos corações desnervados e controles. O estudo hemodinâmico mostrou que a cardiopatia cursa com redução da contratilidade cardíaca.

O sistema de condução e a inervação extrínseca parassimpática do coração foram preservados, uma vez que o ECG de repouso e a resposta a estimulação elétrica do nervo vago foram semelhantes nos dois grupos estudados. Porém, a diminuição do intervalo R-R no ECG do grupo CB denota um sinal indireto de cardiopatia grave.

A análise dos parâmetros hemodinâmicos, principalmente queda do índice cardíaco e da fração de ejeção avaliados pelo volume sistólico, associada à congestão passiva crônica hepática no grupo $\mathrm{CB}$, evidencia insuficiência cardíaca congestiva direita e esquerda. A redução da pressão arterial média (PAM) no grupo CB sugere um quadro de falência cardiocirculatória presente na insuficiência cardíaca descompensada. Não houve diferença estatística na comparação dos parâmetros freqüência cardíaca $(\mathrm{FC})$ e resistência periférica total o que corrobora com a hipótese de que a alteração é na contratilidade miocárdica.

Somente hipóteses podem ser levantadas sobre os mecanismos que reduzem a contratilidade cardíaca após a desnervação do SNIC. É consenso que a insuficiência cardíaca acompanha-se de importante distúrbio no sistema nervoso autonômico. Essa disautonomia na insuficiência cardíaca é, em geral, caracterizada pelo aumento da atividade do sistema nervoso simpático e diminuição da atividade do sistema nervoso parassimpático [14]. Um mecanismo fisiopatológico da falência cardíaca comum aos modelos de laboratório e na população é a ativação neuroendócrina [15], com elevação dos níveis de noradrenalina [16], vasopressina, fator atrial natriurético, endotelina e aumento do sistema renina-angiotensinaaldosterona [17].

Uma forma para estudar a função da inervação intrínseca cardíaca seria avaliar as alterações decorrentes da desnervação na cardiopatia chagásica [14]. Entretanto, a inconveniência que o modelo experimental de desnervação pela Doença de Chagas apresenta se deve às dificuldades da padronização da desnervação e ao fato de que nesta cardiopatia coexistem fenômenos imunológicos e de alterações na microvasculatura [18] que impossibilitam a identificação isolada do papel da desnervação nas alterações morfológicas e funcionais do coração.

Nos modelos de desnervação descritos na literatura como a plexectomia [19], transplante [20], técnica de Randall/ vagotomia [21] e autotransplante [22] apenas a inervação extrínseca foi comprometida em animais e seres humanos. Esses modelos não levam a uma alteração na fisiologia ou morfologia cardíaca. O modelo estudado difere fundamentalmente pelo fato de provocar uma desnervação exclusivamente intrínseca e provocar uma importante alteração na contratilidade cardíaca. 
Neste sentido, a desnervação intrínseca cardíaca se assemelha a cardiopatias dilatadas não isquêmicas, como a cardiopatia chagásica e a diabética. Nessas doenças, existe um desbalanço do sistema nervoso autonômico e a desnervação intrínseca tem, sem dúvida alguma, uma importância capital. Este é o detalhe experimental que faza desnervação como $\mathrm{CB}$ um modelo original. Os nossos resultados abrem amplas perspectivas para estudos futuros sobre o papel dos neurônios intracardíacos na fisiopatologia do coração.

\section{AGRADECIMENTOS}

Paulo B. Évora pelas sugestões inestimáveis, apoio constante e incentivo; J.A.M.Oliveira, S.Zucoloto e H.Salgado por permitirem o uso de seus laboratórios de pesquisa para realização de experimentos; A.Haddad, pelo apoio logístico inusitado; Vani Maria Alves Correia, Isilda Rodrigues Violante e Domingos Soares de Souza Filho pelo apoio técnico.

\section{REFERÊNCIAS BIBLIOGRÁFICAS}

1. Gray AL, Johnson TA, Ardell JL, Massari VJ. Parasympathetic control of the heart. II. A novel interganglionic intrinsic cardiac circuit mediates neural control of heart rate. J Appl Physiol 2004;96:2273-8.

2. Randall DC, Brown DR, McGuirt AS, Thompson GW, Armour JA, Ardell JL. Interactions within the intrinsic cardiac nervous system contribute to chronotropic regulation. Am J Physiol Regul Integr Comp Physiol 2003;285:R1066-75.

3. Lower RR, Stofer RC, Swumway NE. Homovital transplantation of the heart. J Thorac Cardiovasc Surg 1961;41:196-204.

4. Geis WP, Tatooles CJ, Kaye MP, Randall WC. Complete cardiac denervation without transplantation: a simple and reliable technique. J Appl Physiol 1971;30:289-93.

5. Wharton J, Polak JM, Gordon L, Banner NR, Springall DR, Rose $\mathrm{M}$ et al. Immunohistochemical demonstration of human cardiac innervation before and after transplantation. Circ Res 1990;66:900-12

6. Ardell JL. Structure and function of mammalian intrinsic cardiac neurons. In: Armour JA, Ardell JL, eds. Neurocardiology. New York:Oxford University Press;1994.p95-104.

7. Sato A, Yamamoto M, Imamura K, Kashiki Y, Kunieda T, Sakata K. Pathophysiology of aganglionic colon and anorectum: an experimental study on aganglionosis produced by a new method in the rat. J Pediatr Surg 1978;13:399-405.

8. Oliveira JS, Llorach-Velludo MA, Sales-Neto VN. Megacolon in rats. Digestion 1990;45:166-71.
9. Moravec J, Moravec M. Intrinsic nerve plexus of mammalian heart: morphological basis of cardiac rhythmical activity? Int Rev Cytol 1987;106:89-148.

10. Armour JA. Intrinsic cardiac neurones. J Cardiov Electrophisiol 1991;2:331-41.

11. Erbas T, Erbas B, Kabakci G, Aksoyek S, Koray Z, Gedik O Plasma big-endothelin levels, cardiac autonomic neuropathy, and cardiac functions in patients with insulin-dependent diabetes mellitus. Clin Cardiol 2000;23:259-63.

12. Oliveira JS. A natural model of intrinsic heart nervous system denervation: Chagas' cardiopathy. Am Heart Journal 1985;110:1092-8.

13. Garcia SB, Kawasaky MC, Silva JC, Garcia-Rodrigues AC Borelli-Bovo TJ, Iglesias AC et al. Intrinsic myenteric denervation: a new model to increase the intestinal absorptive surface in short-bowel syndrome. J Surg Res 1999;85:200-3.

14. Machado CR, Camargos ER, Guerra LB, Moreira MC. Cardiac autonomic denervation in congestive heart failure: comparison of Chagas' heart disease with other dilated cardiomyopathy. Hum Pathol 2000;31:3-10.

15. McCann SM, Gutkowska J, Antunes-Rodrigues J. Neuroendocrine control of body fluid homeostasis. Braz J Med Biol Res 2003;36:165-81.

16. Cohn JN, Levine TB, Olivari MT, Garberg V, Lura D, Francis GS et al. Plasma norepinephrine as a guide to prognosis in patients with chronic congestive heart failure. N Engl J Med 1984;311:819-23.

17. Fergunson DW, Mark AL. Clinical neurocardiology: role of the autonomic nervous system in clinical heart failure. In: Armour JA, Ardell JL, eds. Neurocardiology. New York:Oxford University Press;1994. p.397-423.

18. Rossi MA, Bestetiti RB. The challenge of chagasic cardiomyopathy: the pathologic roles of autonomic abnormalities, autoimmune mechanisms and microvascular changes and therapeutic implications.Cardiology 1995;86:1-7.

19. Arnulf G. De la section du plexus préaortique: justification et technique. Presse Med 1939;94:1635-41.

20. Fullerton DA, Mitchell MB, McIntyre Jr RC, Brown JM, Meng X, Campbell DN et al. Mechanisms of coronary vasomotor dysfunction in the transplanted heart. Ann Thorac Surg 1994;58:86-92.

21. Randall WC, Kaye MP, Thomas JX, Barber MJ. Intrapericardial denervation of the heart. J Surg Res 1980;29:101-9.

22. Bertrand ME, Lablanche JM, Tilmant PY, Ducloux G Warembourg Jr H, Soots G. Complete denervation of the heart (autotransplantation) for treatment of severe, refractory coronary spasm. Am J Cardiol 1981;47:1375-8. 